\title{
Distribution Amplitudes of Light Mesons and the Photon in the Instanton Model
}

\author{
Alexander E. Dorokhov \\ Bogoliubov Laboratory of Theoretical Physics, \\ Joint Institute for Nuclear Research, 141980 Dubna, Russia
}

Received on 30 October, 2006; revised version received on 17 February, 2007

\begin{abstract}
The leading- and higher-twist distribution amplitudes of the pion, the $\rho$-meson and real and virtual photons are analyzed in the instanton liquid model.
\end{abstract}

Keywords: QCD; Instanton; High energy; Quark; Pion; Photon; Distribution amplitude

\section{INTRODUCTION}

The investigations of hard exclusive processes are essential for our understanding of the internal quark-gluon dynamics of hadrons. Theoretically, such studies are based on the assumption of factorization of dynamics at long and short distances. The short-distance physics is well elaborated by perturbative methods of QCD and depends in particular on the hard subprocesses. The long-distance dynamics is essentially nonperturbative and within the factorization formalism becomes parametrized in terms of hadronic distribution amplitudes (DAs). These nonperturbative quantities are universal and are defined as vacuum-to-hadron matrix elements of particular nonlocal light-cone quark or quark-gluon operators. The evolution of DAs at sufficiently large virtuality $q^{2}$ is controlled by the renormalization scale dependence of the quark bilinear operators within QCD perturbation theory. For the leading-order DAs this dependence is governed by the QCD evolution equations. When the normalization scale goes to infinity the DAs reach an ultraviolet fixed point and are uniquely determined by perturbative QCD. However, the derivation of the DAs themselves at an initial scale $\mu_{0}^{2}$ from first principles is a nonperturbative problem and remains a serious challenge.

Here we present the results $[1,2]$ of the study of the pion, $\rho$-meson and photon DAs in the leading and higher twists at a low-momentum renormalization scale in the gauged non-local chiral quark model [3-5] based on the instanton picture of the QCD vacuum.

\section{DEFINITIONS AND NOTATIONS}

The distribution amplitudes of the mesons or the photon are defined via the matrix elements of quark-antiquark bilinear operators taken between the vacuum and the hadronic state $|h(q)\rangle$ of momentum $q$. It is assumed that the quark and antiquark are separated by the distance $2 z$ and the light-like limit $z^{2} \rightarrow 0$ is taken at a fixed scalar product $q \cdot z$. We use the lightcone expansion of the matrix elements in order to define the $\mathrm{DAs}^{1}$ (only leading twist terms are presented)

$$
\left\langle 0\left|\bar{d}(z) \gamma_{\mu} \gamma_{5}[z,-z] u(-z)\right| \pi^{+}(q)\right\rangle=i \sqrt{2} f_{\pi} q_{\mu} \int_{0}^{1} d x e^{i \xi q \cdot z} \phi_{\pi}^{\mathrm{A}}(x),
$$

\footnotetext{
${ }^{1}$ Our definitions of the photon and $\rho$-meson DAs follow closely the works of Braun, Ball and coauthors [6, 7].
}

$$
\begin{aligned}
& \left\langle 0\left|\bar{q}(z) \sigma_{\mu v}[z,-z] q(-z)\right| \gamma^{\lambda}(q)\right\rangle=i e_{q}\langle 0|\bar{q} q| 0\rangle \chi_{\mathrm{m}} \\
& \cdot f_{\perp \gamma}^{t}\left(q^{2}\right)\left(e_{\mu}^{(\lambda)} q_{v}-q_{\mu} e_{v}^{(\lambda)}\right) \int_{0}^{1} d x e^{i \xi q \cdot z} \phi_{\perp \gamma}\left(x, q^{2}\right) \\
& \left\langle 0\left|\bar{q}(z) \gamma_{\mu}[z,-z] q(-z)\right| \gamma^{\lambda}(q)\right\rangle=e_{q} f_{3 \gamma} f_{\| \gamma}^{v}\left(q^{2}\right) q_{\mu} \\
& \cdot \frac{e^{(\lambda)} \cdot z}{q \cdot z} \int_{0}^{1} d x e^{i \xi q \cdot z} \phi_{\| \gamma}\left(x, q^{2}\right)
\end{aligned}
$$

where $f_{\pi}$ is the pion decay constant, $\langle 0|\bar{q} q| 0\rangle$ is the quark condensate, $\chi_{m}$ is the magnetic susceptibility of the quark condensate, and $f_{3 \gamma}$ is related to the first moment of the magnetic susceptibility. The symbol $[-z, z]$ in the matrix elements denotes the path-ordered gauge link (Wilson line) for the gluon fields between the points $-z$ and $z$. The integration variable $x$ corresponds to the momentum fraction carried by the quark and $\xi=2 x-1$ for the short-hand notation. For a real photon, due to condition $e^{(\lambda)} \cdot z=0$, the structure corresponding to $\phi_{\| \gamma}$ decouples. The DAs $\phi_{\perp \rho}(x)$ and $\phi_{\| \rho}(x)$ for the $\rho$-meson state $\left|\rho^{\lambda}(q)\right\rangle$ are defined in analogy with the photon cases (2) and (3) with the mass-shell condition $q^{2}=-M_{\rho}^{2}$.

\section{INSTANTON-MOTIVATED NONLOCAL CHIRAL QUARK MODEL}

In the one loop approximation the quark model evaluation of the distribution function $\phi_{h, J}(x)$ of hadron $h$ corresponding to projection $J$ is given schematically as [8]

$$
N_{h, J} \phi_{h, J}(x)=-i N_{c} \int d \tilde{k} \delta(k \cdot n-x) \operatorname{Tr}\left[\Gamma_{J} S(k) \Gamma_{h} S(k-q)\right],
$$

where the quark propagator has the form

$$
S(p)=\frac{1}{\widehat{p}-M(p)+i \varepsilon}, \quad M(p)=M_{0} f^{2}\left(p^{2}\right),
$$

with the dynamical quark mass $M(p)$ expressed via the function $f(p)$ defining the nonlocal properties of the QCD vacuum [9]. $\Gamma_{h}$ are the vertices defining the hadron state

$$
\begin{aligned}
& \Gamma_{\pi}\left(k, k^{\prime}\right)=\gamma_{5} f(k) f\left(k^{\prime}\right), \quad \Gamma_{\rho}^{\mu}\left(k, k^{\prime}\right)=\gamma_{\mu}^{\perp} f_{V}(k) f_{V}\left(k^{\prime}\right), \\
& \Gamma_{\gamma}^{\mu}\left(k, k^{\prime}\right)=\gamma_{\mu}-\left(k+k^{\prime}\right)_{\mu} M_{k, k^{\prime}}^{(1)},
\end{aligned}
$$


and $\Gamma_{J}$ is the projection operator corresponding to a definite twist. Here and below, the notation

$$
M^{(1)}\left(k, k^{\prime}\right)=\frac{M(k)-M\left(k^{\prime}\right)}{k^{2}-k^{\prime 2}}
$$

is used. The nonlocal functions are chosen in the Gaussian form

$$
f(p)=f_{V}(p)=\exp \left(-\frac{p^{2}}{\Lambda^{2}}\right),
$$

with $p$ denoting the Euclidean momentum, resembling the fact that the instanton field is convenient to express in the axial gauge. As the model parameters we take the values fixed in [10]

$$
M_{0}=240 \mathrm{MeV}, \Lambda=1110 \mathrm{MeV} \text {. }
$$

The distribution amplitudes of the pion and the real photon

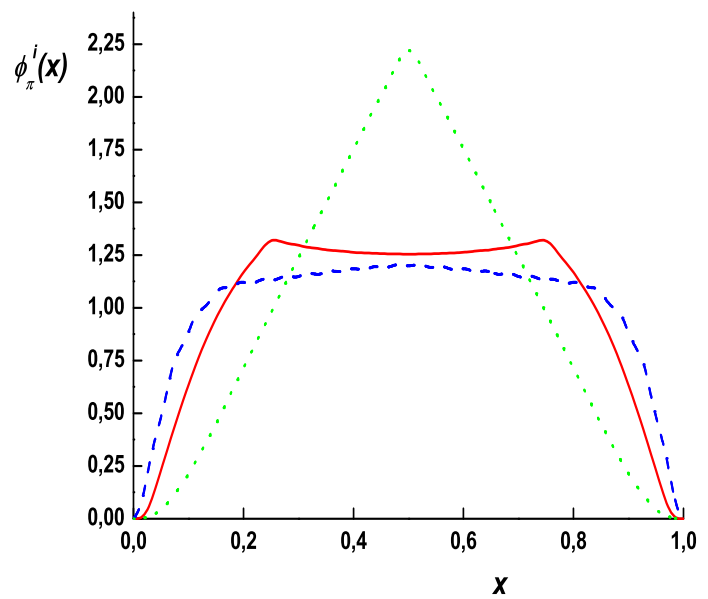

FIG. 1: Pion distribution amplitudes: twist-2 axial-vector (solid line), twist-3 pseudoscalar (short-dashed) and tensor (dotted) projections, given at the quark model scale.

calculated in the instanton model in the chiral limit may be cast in a closed form. It is convenient to introduce notations for the integration variables $(\bar{x}=1-x)$

$$
\begin{aligned}
u_{+} & =u-i \lambda x, \quad u_{-}=u+i \lambda \bar{x}, \quad M_{ \pm}=M\left(u_{ \pm}\right), \\
D(u) & =u+M^{2}(u), \quad D_{ \pm}=D\left(u_{ \pm}\right) .
\end{aligned}
$$

Then one gets the expressions

$$
\begin{gathered}
\phi_{\pi}^{A}(x)=\frac{1}{f_{\pi}^{2}} \frac{N_{c}}{4 \pi^{2}} \int_{0}^{\infty} d u \int_{-\infty}^{\infty} \frac{d \lambda}{2 \pi} \frac{f_{+} f_{-}}{D_{+} D_{-}}\left(x M_{-}+\bar{x} M_{+}\right) \\
\phi_{\perp \gamma}\left(x, q^{2}=0\right)=\frac{1}{|\langle\bar{q} q\rangle| \chi_{\mathrm{m}}} \frac{N_{c}}{4 \pi^{2}}\left[\Theta(\bar{x} x) \int_{0}^{\infty} d u \frac{M(u)}{D(u)}-\right. \\
\left.-\int_{0}^{\infty} d u \int_{-\infty}^{\infty} \frac{d \lambda}{2 \pi} \frac{M_{+} M_{-}}{D_{+} D_{-}} M^{(1)}\left(u_{+}, u_{-}\right)\right] \\
\phi_{\| \gamma}\left(x, q^{2}=0\right)=\Theta(\bar{x} x) .
\end{gathered}
$$

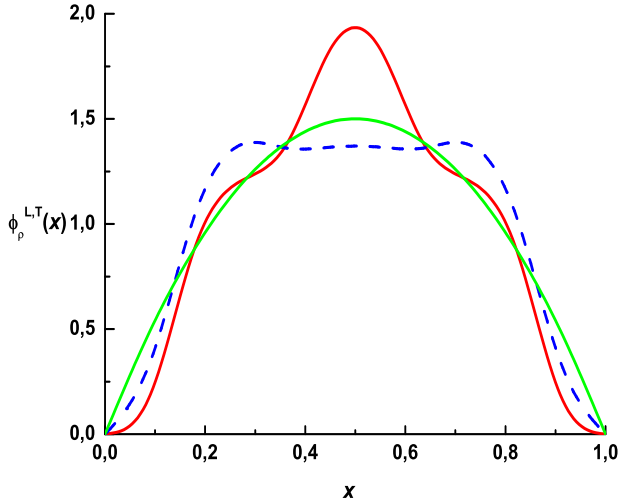

FIG. 2: $\rho$-meson twist-2 distribution amplitudes: transverse (solid line) and longitudinal (dashed) projections. The third line is distribution amplitude at asymptotic scale.

The DAs are scale dependent quantities and the above expressions correspond to the low momentum scale $\mu_{0}$ typical for the instanton model. For the instanton model it is estimated to be $\mu_{0}=530 \mathrm{MeV}$ [11]. The parameters entering the normalization coefficients are given by

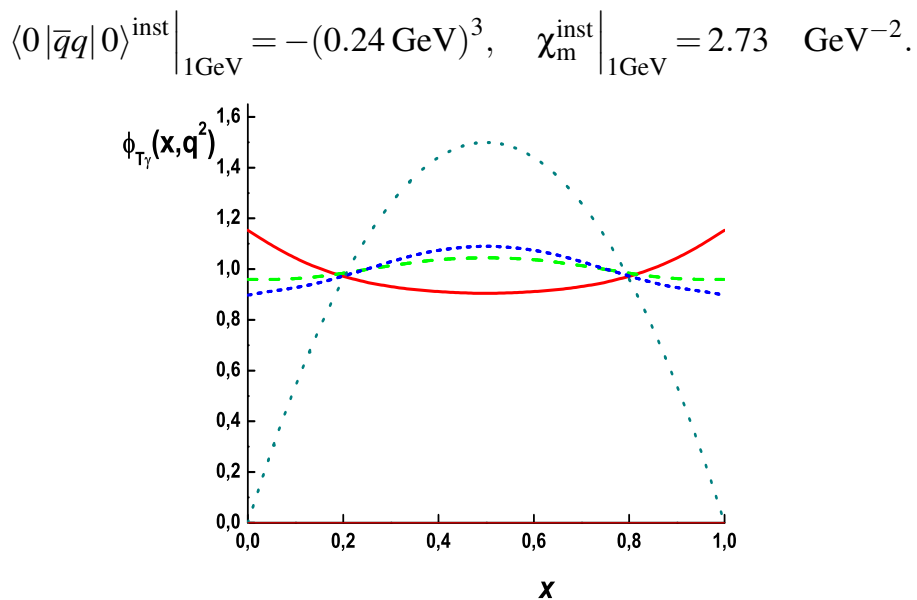

FIG. 3: Dependence of the twist-2 tensor component of the photon DA on transverse momentum squared $\left(q^{2}=0.25 \mathrm{GeV}^{2}\right.$ solid line, $q^{2}=0 \mathrm{GeV}^{2}$ dashed line, $q^{2}=-0.09 \mathrm{GeV}^{2}$ short-dashed line, asymptotic DA - dotted line) given at the quark model scale.

The results of calculations are shown in Figs. 1-4. They correspond to the low momentum scale $\mu_{0}$ and need to be evolved to higher momenta scales in order to compare with the experimentally available information. The DA at asymptotic scales $\mu_{\mathrm{as}}=\infty$ is also presented.

\section{DISCUSSION}

Within the instanton model the leading twist pion DA has been found in [12-14], the twist-3 and twist-4 DAs in [12] and [1], respectively, and the photon DAs up to twist-4 expansion in [2]. Recently there have been published several papers [15-18], where in nonlocal models, similar to the one 


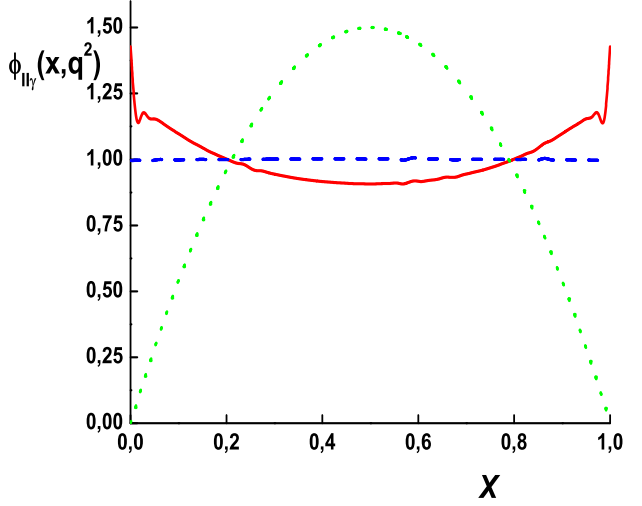

FIG. 4: Same as Fig. 3 for the twist-2 vector component of the photon DA.

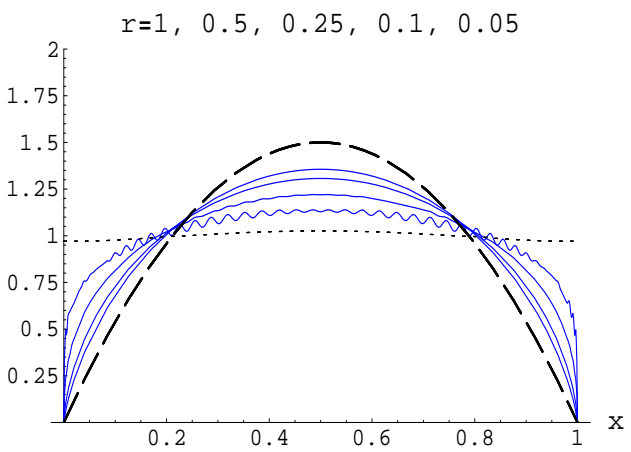

FIG. 5: The LO ERBL evolution of the nonlocal model predictions for the leading-twist tensor projection of the real photon DA $\phi_{\perp \gamma}^{(t)}\left(x, q^{2}\right)$. The dashed lines show the asymptotic DA, $6 x(1-x)$. Initial conditions, indicated by dotted lines, are evaluated in the nonlocal quark model at the initial scale $\mu^{\text {inst }}=530 \mathrm{MeV}$. The solid lines correspond to evolved DA'a at scales $Q=1,2.4,10$, and $1000 \mathrm{GeV}$. The corresponding values of the evolution ratio $r$ are given in the figures. considered above, the distribution functions for pion and photon were treated inconsistently. Indeed, the typical expression (4) defining the distribution functions has two parts. The first is coming from the soft hadronic vertices (6), and the second refers to the operators of definite twist which are responsible for the power corrections in the hard subprocess. It is well known, that for example, the leading twist operators, entering the pion distribution amplitude and distribution function, are given by $\gamma_{\mu} \gamma_{5}$ and $\gamma_{\mu}$, correspondingly. Nevertheless, the authors of [15-17] included additional terms proportional to $\left(k+k^{\prime}\right)_{\mu}$, where $k$ and $k^{\prime}$ are incoming and outgoing momenta of a quark. These additions in [15-17] modify the known results of the leading twist calculations. However, it is evident, see for example [1], that these additional terms contribute to twist-4 distributions and do not touch the leading twist-2 distributions. From the other side, the full vertex including the local and nonlocal pieces is important in the soft hadronic part and has not to be neglected as it was done in $[14,18]$ considering the photon distribution amplitudes.

\section{CONCLUSIONS}

The instanton model of QCD vacuum is a realistic tool to obtain the nonperturbative properties of hadrons in terms of parameters characterizing the vacuum. All hadron DAs are suppressed at the bound of the kinematical interval, due to the nonlocality of the wave function of the hadrons, while the photon DAs are non vanishing there. By applying the QCD evolution, the photon DAs become immediately zero at the edge points of the $x$-interval. Nevertheless, the photon DAs are always wider than the asymptotic distribution. The first experimental results on the direct measurements of the pion and photon DAs are discussed in [19, 20].

\section{Acknowledgements}

The author thanks the organizers for the very fruitful meeting and the Russian Foundation for Basic Research projects No. 04-02-16445, Scient. School grant 4476.2006.2 and the JINR Heisenberg-Landau program for partial support.
[1] A. E. Dorokhov, JETP Lett. 77, 63 (2003).

[2] A. E. Dorokhov, W. Broniowski, and E. Ruiz Arriola, Phys. Rev. D 74, 054023 (2006).

[3] R. D. Bowler and M. C. Birse, Nucl. Phys. A 582, 655 (1995).

[4] I. V. Anikin, A. E. Dorokhov, and L. Tomio, Phys. Part. Nucl. 31, 509 (2000).

[5] A. E. Dorokhov and W. Broniowski, Eur. Phys. J. C 32, 79 (2003).

[6] P. Ball, V. M. Braun, Y. Koike, and K. Tanaka, Nucl. Phys. B 529, 323 (1998).

[7] P. Ball, V. M. Braun, and N. Kivel, Nucl. Phys. B 649, 263 (2003).

[8] A. E. Dorokhov and L. Tomio, Phys. Rev. D 62, 014016 (2000).

[9] A. E. Dorokhov, S. V. Esaibegian, and S. V. Mikhailov, Phys. Rev. D 56, 4062 (1997).
[10] A. E. Dorokhov, Phys. Rev. D 70, 094011 (2004).

[11] A. E. Dorokhov, Eur. Phys. J. C 42, 309 (2005).

[12] S. V. Esaibegian and S. N. Tamarian, Sov. J. Nucl. Phys. 51, 310 (1990).

[13] A. E. Dorokhov, Nuovo Cim. A 109, 391 (1996).

[14] V. Y. Petrov, M. V. Polyakov, R. Ruskov, C. Weiss, and K. Goeke, Phys. Rev. D 59, 114018 (1999).

[15] S. Noguera and V. Vento, Eur. Phys. J. A 28, 227 (2006).

[16] S. I. Nam, H. C. Kim, A. Hosaka, and M. M. Musakhanov, Phys. Rev. D 74, 014019 (2006).

[17] S. I. Nam and H. C. Kim, arXiv:hep-ph/0609267.

[18] R. Yu, J. Liu, and K. Zhu, Phys. Rev. D 73, 045002 (2006).

[19] E791, E. M. Aitala et al., Phys. Rev. Lett. 86, 4773 (2001).

[20] ZEUS, J. M. Ukleja, Eur. Phys. J. C 33, s506 (2004). 